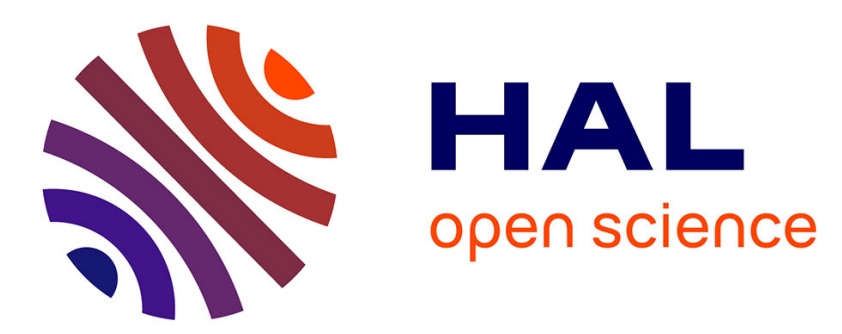

\title{
Connected components of PGL(2,R)-Representation Spaces of Non-Orientable Surfaces
}

Frédéric Palesi

\section{To cite this version:}

Frédéric Palesi. Connected components of PGL(2,R)-Representation Spaces of Non-Orientable Surfaces. Geometry, Topology and Dynamics of Character Varieties, 23, World Scientific Publishing Co. Pte. Ltd., pp. 349, 2012, Lecture Notes Series, Institute for Mathematical Sciences, National University of Singapore, 981-4401-35-8. 10.1142/9789814401364_0008 . hal-01151141

\section{HAL Id: hal-01151141 \\ https://hal.science/hal-01151141}

Submitted on 12 May 2015

HAL is a multi-disciplinary open access archive for the deposit and dissemination of scientific research documents, whether they are published or not. The documents may come from teaching and research institutions in France or abroad, or from public or private research centers.
L'archive ouverte pluridisciplinaire HAL, est destinée au dépôt et à la diffusion de documents scientifiques de niveau recherche, publiés ou non, émanant des établissements d'enseignement et de recherche français ou étrangers, des laboratoires publics ou privés. 


\title{
CONNECTED COMPONENTS OF PGL(2, R)-REPRESENTATION SPACES OF NON-ORIENTABLE SURFACES
}

\author{
FRÉDÉRIC PALESI
}

\begin{abstract}
The Teichmüller space of a surface naturally embeds as a connected component in the moduli space of representations from the fundamental group of the surface into the group of isometries of the hyperbolic plane. We present invariants that distinguish all the connected components of the space of representations. This allows us to compute the number of connected components of these spaces both in the orientable and in the non-orientable case.
\end{abstract}

\section{INTRODUCTION}

Let $G$ be a Lie group and $S$ be a closed surface (orientable or not) with Euler characteristic $\chi(S)<0$. Its fundamental group is denoted by $\pi=\pi_{1}(S)$ with respect to a base point. The representation space $\operatorname{Hom}(\pi, G)$ is the set of all homomorphisms $\phi: \pi \rightarrow G$. The group $G$ acts by conjugation on the representation space, and the quotient of $\operatorname{Hom}(\pi, G)$ by this action is called the moduli space of representations

$$
\operatorname{Hom}\left(\pi_{1}(S), G\right) / G=\mathcal{X}(S, G)
$$

Moduli spaces of representations appear naturally in many contexts, depending on the group $G$. For example we can identify the moduli space as the set of isomorphism classes of flat $G$-bundles on the surface. These spaces admit a natural action of the mapping class group exhibiting very interesting dynamics $([8,14])$.

When $G$ is non-compact, the moduli space $\mathcal{X}(S, G)$ contains contractible components which correspond to locally homogeneous geometric structures on the surface (see [9] for a survey on such structures). In particular, when $G=\operatorname{Isom}\left(\mathbb{H}^{2}\right)$ is the group of isometries of the hyperbolic plane, the related geometric structures on the surface will be hyperbolic. Other geometric structures can be recovered from other groups $G$ such as complex projective structures when $G=\operatorname{PSL}(2, \mathbb{C})($ see $[5])$, or convex projective structures when $G=\operatorname{PSL}(3, \mathbb{R})($ see $[4])$. 
The Teichmüller space $\mathcal{T}(S)$ is equivalent by the uniformization theorem to the set of equivalence classes of complete hyperbolic structures on $S$. There is an isomorphism between $\operatorname{Isom}\left(\mathbb{H}^{2}\right)$ and the Lie group $\operatorname{PGL}(2, \mathbb{R})$ through the identification of $\mathbb{H}^{2}$ with the upper-half plane. When the surface $S$ is given a hyperbolic structure, the holonomy map gives a representation of $\pi$ into $\operatorname{PGL}(2, \mathbb{R})$. This representation is only well defined up to conjugation by an element of $\operatorname{PGL}(2, \mathbb{R})$ (corresponding to a choice of a base point for $\pi_{1}(S)$ ). Hence we have a well defined map

$$
\text { hol }: \mathcal{T}(S) \longrightarrow \mathcal{X}(S, \operatorname{PGL}(2, \mathbb{R})) \text {. }
$$

This map is a proper embedding, and its image is exactly the set of $\operatorname{PGL}(2, \mathbb{R})$-equivalence classes of discrete and faithful representations. The subset of such representations is well-understood inside the moduli space as shown in the following theorem (see [7]):

Theorem 1. Let $S$ be a closed surface (orientable or not). The set of $\operatorname{PGL}(2, \mathbb{R})$-equivalence classes of discrete and faithful representations is a connected component of the moduli space $\mathcal{X}(S, \operatorname{PGL}(2, \mathbb{R}))$.

Obviously, representation spaces and moduli spaces contain more representations than the discrete and faithful ones, so these spaces have more than one connected component. A natural question is to find some topological invariants that allow one to distinguish the connected components and hence compute the number of components.

For representation $\phi: \pi_{1}(S) \rightarrow \operatorname{PGL}(2, \mathbb{R})$, a classical invariant is the so-called Euler class that is associated to the circle bundle over the surface defined by $\phi$. This invariant can be defined in several ways, and the main objective of this note is to give a simple construction valid both in the orientable and the non-orientable case. And we give some insight into how this invariant distinguishes the connected components of the representation space.

In the classical case of orientable surfaces, the holonomy of a hyperbolic structure lies in the group of orientation-preserving isometries $\operatorname{Isom}^{+}\left(\mathbb{H}^{2}\right)$, which is identified with the group $\operatorname{PSL}(2, \mathbb{R})$.

In Section 2, we will give some basic properties of the group $\operatorname{PSL}(2, \mathbb{R})$ and its universal cover. In particular, we will study the behavior of the map corresponding to the relation of the fundamental group of the surface. This is the key ingredient to define a topological invariant using a simple algorithm.

In Section 3, we first define this invariant for closed orientable surfaces, and then generalize it to orientable surfaces with boundary. This invariant is all that we need to distinguish the connected components 
of the representation space $\operatorname{Hom}\left(\pi_{1}(\Sigma), \operatorname{PSL}(2, \mathbb{R})\right)$, and give a characterization for the discrete and faithful representations. In [7], Goldman proves the following :

Theorem 2. Let $\Sigma$ be a closed orientable surface with $\chi(\Sigma)<0$. The representation space $\operatorname{Hom}(\pi, \operatorname{PSL}(2, \mathbb{R}))$ has $2|\chi(\Sigma)|+1$ connected components, indexed by the Euler class $\chi(\Sigma) \leq e \leq-\chi(\Sigma)$.

Moreover, a representation is discrete and faithful if and only if it is in the maximal components satisfying $|e|=|\chi(\Sigma)|$.

We give only some elements of the proof of this result and refer to Goldman's original paper for a complete proof. This question has also been generalized and solved for a variety of other Lie groups, for example when $G=\operatorname{PSL}(n, \mathbb{R})$ (see $[10]), G=\operatorname{Sp}(2 n, \mathbb{R})$ (see $[2,6])$, or when the symmetric space associated to $G$ is Hermitian (see [17]).

In Section 4, we extend the construction of the topological invariant defined in Section 3 to the case of a non-orientable surface $N$. In this case, the invariant is only well defined in $\mathbb{Z} / 2 \mathbb{Z}$ and the representation space $\operatorname{Hom}\left(\pi_{1}(N), \operatorname{PSL}(2, \mathbb{R})\right)$ has only 2 connected components. These components do not contain any holonomy representations for hyperbolic structures (see [15]).

In this case, it is natural to consider not only orientation-preserving isometries, but also orientation reversing isometries. Hence, the interesting representation space is $\operatorname{Hom}(\pi, \operatorname{PGL}(2, \mathbb{R}))$. For representation into $\operatorname{PGL}(2, \mathbb{R})$, we have to define another invariant that takes values in $(\mathbb{Z} / 2 \mathbb{Z})^{k}$, and then understand the set of representations for each value of this invariant. The main result of [16] is the following :

Theorem 3. Let $N$ be a closed non-orientable surface with $\chi(N)<0$, then the representation space $\operatorname{Hom}(\pi, \operatorname{PGL}(2, \mathbb{R}))$ has $2^{3-\chi(n)}-2 \chi(N)-$ 1 connected components indexed by two obstruction classes.

\section{The GRoup PSL $(2, \mathbb{R})$}

The fundamental group of a surface is a finitely presented group with $p$ generators and $q$ relations. So for any Lie group $G$ the representation space $\operatorname{Hom}(\pi, G)$ can be identified with the subset of $G^{p}$ defined by $q$ equations in words in $G$, corresponding to the $q$ relations. So to understand the representation space, we study the properties of $G$ and of the map

$$
\begin{aligned}
G^{p} & \longrightarrow G^{q} \\
\left(g_{1}, \ldots, g_{p}\right) & \longmapsto\left(R_{1}\left(g_{1}, \ldots, g_{p}\right), \ldots, R_{q}\left(g_{1}, \ldots, g_{p}\right)\right)
\end{aligned}
$$


where the $R_{i}$ are the $q$ relations, each in $p G$-valued unknowns.

In the following section, we shall note $\widehat{G}=\operatorname{SL}(2, \mathbb{R})$ and $G=$ $\operatorname{PSL}(2, \mathbb{R})=\widehat{G} /\{ \pm I\}$. We see that $\widehat{G}$ is a double cover of $G$.

2.1. Universal cover. The standard polar decomposition of $\operatorname{SL}(2, \mathbb{R})$ allows one to decompose any $A \in \widehat{G}$ in a unique way into $A=R \cdot P$, with $R \in \mathrm{SO}(2)$ and $P \in \mathrm{Sym}$, the group of symmetric positive definite matrices of determinant 1 . The group Sym being contractible, we have a natural deformation retraction of $G$ onto the circle $\operatorname{SO}(2) \simeq \mathbb{S}^{1} \simeq$ $\mathbb{R} / \mathbb{Z}$

So, the fundamental group of $G$ is $\pi_{1}(G) \simeq \pi_{1}\left(\mathbb{S}^{1}\right) \simeq \mathbb{Z}$. Let $\widetilde{G}$ be the universal cover of $G$ and $p: \widetilde{G} \rightarrow G$, the covering map. The center of $\widetilde{G}, Z(\widetilde{G})=\operatorname{ker}(p)$ is isomorphic to $\mathbb{Z}$ and $G \simeq \widetilde{G} / \mathbb{Z}$. We denote by $z$ a generator of $Z(\widetilde{G})$ so that $Z(\widetilde{G})=\langle z\rangle$.

Two lifts of the same element will differ by an element of $Z(\widetilde{G})$. In other words, if $A, B \in \widetilde{G}$ satisfy $p(A)=p(B)$, then there exists $n \in \mathbb{Z}$ such that $A=z^{n} B$.

The group $\widehat{G}$ is a connected 2-fold cover of $G$, and thus $\widetilde{G}$ is also the universal cover of $\widehat{G}$. We have a canonical identification $G=\widetilde{G} /\left\langle z^{2}\right\rangle$.

2.2. Classification of elements. The orientation-preserving isometries can be classified through their number of fixed points on the boundary. An element $f$ in $\operatorname{Isom}^{+}(\mathbb{H})^{2} \backslash\{I d\}$, is said to be elliptic (resp. parabolic and hyperbolic) when $f$ has no fixed points on the boundary $\partial \mathbb{H}^{2}$ (resp. one and two fixed points)

This can be interpreted in terms of the trace of corresponding elements of $\operatorname{PSL}(2, \mathbb{R})$. Notice that only the absolute value of the trace is well defined in $G$. An element $A$ in $G \backslash\{I\}$, is elliptic (resp. parabolic and hyperbolic) when $|\operatorname{tr}(A)|<2$ (resp. when $|\operatorname{tr}(A)|=2$ or $|\operatorname{tr}(A)|>2$.

Lifts of elliptic (resp. parabolic, hyperbolic) elements in the universal cover $\widetilde{G}$ will also be called elliptic (resp. parabolic, hyperbolic) and we denote by $\mathcal{E}, \mathcal{P}$ and $\mathcal{H}$ the corresponding subsets of $\widetilde{G}$. These subsets decompose $\widetilde{G}$ into infinitely many disjoint subsets, indexed by integers $\mathbb{Z}$ corresponding to different copies of $G$ inside $\widetilde{G}$, according to Figure 1.

We can distinguish these regions using a simple invariant of $A \in \widetilde{G}$. The action of $G$ on $\mathbb{H}^{2}$ extends to an action on the circle at infinity $\partial \mathbb{H}^{2} \simeq \mathbb{S}^{1}$. This action lifts to an action of $\widetilde{G}$ on $\widetilde{\mathbb{S}^{1}} \simeq \mathbb{R}$. Hence for 


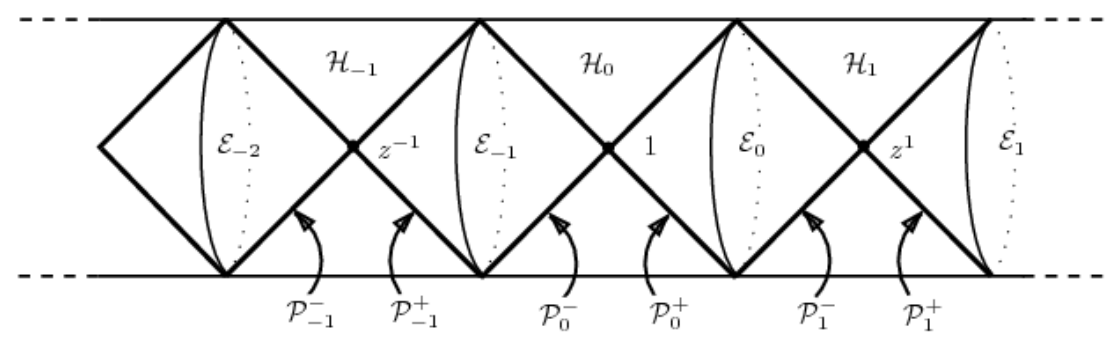

Figure 1. Domains of $\widetilde{G}$

any element in $\widetilde{G}$ we can define

$$
\begin{aligned}
& \underline{m} A=\min \{A \cdot x-x \mid x \in \mathbb{R}\}, \\
& \bar{m} A=\max \{A \cdot x-x \mid x \in \mathbb{R}\} .
\end{aligned}
$$

Using these invariants, we can give the following characterizations [11]:

$$
\begin{aligned}
& A \in \mathcal{E}_{i}\Longleftrightarrow[\underline{m} A, \bar{m} A] \subset] i, i+1[, \\
& A \in \mathcal{H}_{i}\Longleftrightarrow i \in] \underline{m} A, \bar{m} A[, \\
& A \in \mathcal{P}_{i}^{+} \Longleftrightarrow \underline{m} A=i<\bar{m} A, \\
& A \in \mathcal{P}_{i}^{-} \Longleftrightarrow \underline{m} A<i=\bar{m} A .
\end{aligned}
$$

There are some direct consequences of these formulas, that are important to understand the case of surfaces with Euler characteristic -1. For example, we have the following proposition (see [7]):

Proposition 1. If $A, B \in \mathcal{H}_{0}$ and $A B \in \mathcal{H}_{i}$ then $i \in\{-1,0,1\}$.

We will see in Section 3 how this will be useful to understand the case of the pair-of-pants.

2.3. The commutator map. The fundamental group of an orientable surface of genus $g$ is given by :

$$
\pi_{1}\left(\Sigma_{g}\right)=\left\langle X_{1}, Y_{1}, \ldots, X_{g}, Y_{g} \mid \prod_{1}^{g}\left[X_{i}, Y_{i}\right]\right\rangle .
$$

Let $A_{1}, B_{1}, \ldots, A_{g}, B_{g}$ be elements of $G$ and choose arbitrary lifts $\widetilde{A_{1}}, \ldots, \widetilde{B_{g}}$ in $\widetilde{G}$. The element defined by :

$$
\widetilde{R_{g}}\left(A_{1}, \ldots, B_{g}\right)=\left[\widetilde{A_{1}}, \widetilde{B_{1}}\right] \cdots\left[\widetilde{A_{g}}, \widetilde{B_{g}}\right],
$$

is independent of the chosen lifts. Indeed two different lifts differ by a central element of $Z(\widetilde{G})$ which will cancel out in $\widetilde{R}$. For example, 
assume that we have two different lifts ${\widetilde{A_{1}}}^{\prime}=g \widetilde{A_{1}}$ where $g \in Z(\widetilde{G})$ then

$$
\begin{aligned}
\widetilde{R}_{g}^{\prime} & =\left[{\widetilde{A_{1}}}^{\prime}, \widetilde{B_{1}}\right] \cdots\left[\widetilde{A_{g}}, \widetilde{B_{g}}\right]=\left(\widetilde{g A_{1}}\right) \cdot \widetilde{B_{1}} \cdot\left({\widetilde{A_{1}}}^{-1} g^{-1}\right) \cdot{\widetilde{B_{1}}}^{-1} \cdots\left[\widetilde{A_{g}}, \widetilde{B_{g}}\right] \\
& =g g^{-1} \cdot\left[\widetilde{A_{1}}, \widetilde{B_{1}}\right] \cdots\left[\widetilde{A_{g}}, \widetilde{B_{g}}\right]=\widetilde{R_{g}}\left(A_{1}, \ldots, B_{g}\right) .
\end{aligned}
$$

So we can define the map

$$
\widetilde{R_{g}}: G^{2 g} \longrightarrow \widetilde{G}
$$

When $g=1$, the image of the commutator map $\widetilde{R_{1}}$ can be explicitely described using the following proposition (see [7]).

Proposition 2. If $\widetilde{R_{1}}(A, B)$ is in $\mathcal{H}_{i}$ then $i \in\{-1,0,1\}$.

We will see in Section 3 how this will be useful to understand the case of the one-holed torus.

2.4. The square map. The fundamental group of a non-orientable surface of genus $k$ is given by

$$
\pi_{1}\left(N_{k}\right)=\left\langle X_{1}, \ldots, X_{k} \mid X_{1}^{2} \cdots X_{k}^{2}\right\rangle .
$$

Let $A_{1}, \ldots, A_{k}$ be elements of $G$ and choose arbitrary lifts $\widetilde{A_{1}}, \ldots, \widetilde{A_{k}}$ in $\widetilde{G}$. The element defined by

$$
Q_{k}\left(A_{1}, \ldots, A_{k}\right)=\left(\widetilde{A_{1}}\right)^{2} \cdots\left(\widetilde{A_{k}}\right)^{2},
$$

is not independent of the chosen lifts. For example, assume that we have two different lifts $\widetilde{A_{1}}=g \widetilde{A_{1}}$ where $g \in Z(\widetilde{G})$ then

$$
Q_{k}^{\prime}=\left(\widetilde{A_{1}}\right)^{2} \cdots\left(\widetilde{A_{k}}\right)^{2}=g^{2}\left(\widetilde{A_{1}}\right)^{2} \cdots\left(\widetilde{A_{k}}\right)^{2}=g^{2} Q_{k}
$$

However, we see that this gives a well defined element of $\widetilde{G} /<z^{2}>=$ $\widehat{G}$, hence this defines a continuous map :

$$
Q_{k}: G \longrightarrow \widehat{G} \text {. }
$$

Using this, we can define the corresponding lifted square map $\widetilde{Q_{k}}$ : $\widetilde{G}^{k} \longrightarrow \widetilde{G}$.

When $k=1$, the image of $Q_{1}$ is easily computed (see [15]) to be :

$$
\operatorname{Im}\left(Q_{1}\right)=J=\{K \in \mathrm{SL}(2, \mathbb{R}) \mid \operatorname{tr}(K)>-2\} \cup\{I\} .
$$

Hence, the image of the lifted square map is :

$$
\operatorname{Im}\left(\widetilde{Q_{1}}\right)=\mathcal{J}=\widetilde{G} \backslash\left(\bigcup_{k \in \mathbb{Z}} \mathcal{H}_{2 k+1} \cup \mathcal{P}_{2 k+1}\right)
$$


We will see in Section 4 how this will be useful to understand the case of one-holed projective plane.

\section{Components For orientable surfaces}

In this section, we give a simple algorithm to compute the Euler class of a representation $\phi: \pi \rightarrow G$ when the surface is orientable and $G=\operatorname{PSL}(2, \mathbb{R})$.

3.1. A topological invariant. Let $\pi=\pi_{1}\left(\Sigma_{g}\right)$ be the fundamental group of the closed orientable surface of genus $g$ and let $\phi$ be an element of $\operatorname{Hom}(\pi, G)$. The relation of the fundamental group implies that the element $\widetilde{R}\left(\phi\left(X_{1}\right), \ldots, \phi\left(Y_{g}\right)\right)$ defined by $(2.3)$ is an element $\operatorname{ker}(p)=\mathbb{Z}$, i.e. there exists $n \in \mathbb{Z}$ such that

$$
\widetilde{R}\left(\phi\left(X_{1}\right), \ldots, \phi\left(Y_{g}\right)\right)=z^{n} .
$$

We define $o_{2}(\phi)=n \in \mathbb{Z}$ and call this number the second obstruction class of the representation $\phi$. (We shall see the first obstruction class in the last section). This defines a map :

$$
o_{2}: \operatorname{Hom}(\pi, \operatorname{PSL}(2, \mathbb{R})) \longrightarrow \mathbb{Z} .
$$

This map is continuous and takes values in a discrete set, so $O_{2}$ is constant on connected components of the representation space.

We can relate this invariant with other classical invariants, such as the characteristic class $w_{2}\left(E_{\phi}\right)$ of the flat $G$-bundle $E_{\phi} \rightarrow \Sigma$, the Euler class $e(\phi)$ of the associated circle bundle over $\Sigma$, or also the Toledo invariant $T(\phi)$ of the representation. All these invariants coincide when $G=\operatorname{PSL}(2, \mathbb{R})$. However, in higher dimension they give different generalizations. For precise definitions and relations between these invariants, we refer to [3].

3.2. Milnor-Wood inequality and a result of Goldman. A circle bundle over $\Sigma$ is simply a fiber bundle $E \rightarrow \Sigma$ whose fibers are isomorphic to $\mathbb{S}^{1}$. A representation $\rho: \pi(S) \rightarrow \operatorname{PSL}(2, \mathbb{R})$ defines a circle bundle over $\Sigma$ through the embedding $G \hookrightarrow \operatorname{Homeo}\left(\mathbb{S}^{1}\right)$ defined by the action of $G$ on $\partial \mathbb{H}^{2}$.

Consider the free product $P=\widetilde{\Sigma} \times \mathbb{S}^{1}$ and the action of $\pi_{1}(\Sigma)$ on $P$ given by :

$$
\begin{aligned}
\pi_{1}(\Sigma) \times\left(\widetilde{\Sigma} \times \mathbb{S}^{1}\right) & \longrightarrow \widetilde{\Sigma} \times \mathbb{S}^{1} \\
\gamma \cdot(x, \theta) & \longmapsto(\gamma \cdot x, \rho(\gamma) \cdot \theta)
\end{aligned}
$$


where the action of $\pi_{1}(\Sigma)$ on $\widetilde{\Sigma}$ is by deck transformations. The quotient of $P$ by this action defines a circle bundle over the surface $S$

$$
E_{\rho}=\left(\widetilde{\Sigma} \times \mathbb{S}^{1}\right) / \pi_{1}(\Sigma),
$$

since the action on the first factor is free. The Euler class of the representation, denoted $e(\rho) \in \mathbb{Z}$ is defined to be the evaluation of the characteristic class $e\left(E_{\rho}\right)$ of the circle bundle on the fundamental cycle $[\Sigma]$. We have $e(\rho)=o_{2}(\rho)$.

Euler classes of circles bundles were studied by Milnor and Wood $[12,13]$ who established the so-called Milnor-Wood inequality, stating that the Euler class of an orientable circle bundle $E$ over $\Sigma$ is bounded by the Euler characteristic of $S$, namely

$$
|e(E)| \leq|\chi(\Sigma)|
$$

Hence the Euler class $e(\rho)$ of a representation can only take $2|\chi(\Sigma)|+$ 1 values, corresponding to the integers contained in the interval $[\chi(\Sigma),-\chi(\Sigma)]$. The Theorem 2 establishes the converse result and we can reformulate it as :

Theorem 4. Let $\Sigma$ be an orientable closed surface with $\chi(\Sigma)<0$, for all $n \in \mathbb{Z}$, if $|n| \leq|\chi(\Sigma)|$ then $e^{-1}(n)$ is non-empty and connected.

The proof of the theorem goes by induction on smaller surfaces that are necessarily surfaces with boundary. So next, we define the invariant in this case and generalize Theorem 2 for surfaces with boundary.

3.3. Surfaces with boundary. For surfaces with boundary, we need to be a little more precise in the definition of the obstruction class. Let $\Sigma=\Sigma_{(g, m)}$, the genus $g$ surface with $m \geq 1$ boundary components denoted $\partial_{1} \Sigma, \ldots, \partial_{m} \Sigma$. Its fundamental group has the following presentation :

$$
\pi_{1}(\Sigma)=\left\langle X_{1}, Y_{1}, \ldots, X_{g}, Y_{g}, C_{1}, \ldots, C_{m} \mid \prod_{1}^{g}\left[X_{i}, Y_{i}\right] \cdot C_{1} \cdots C_{m}\right\rangle .
$$

where, $C_{i}$ corresponds to the boundary circle $\partial_{i} \Sigma$. It is clear that $\pi_{1}(\Sigma)$ is isomorphic to the free group $F_{N}$ in $N=2 g+m-1$ generators, because we can express one of the $C_{i}$ as a word in the other generators.

In this case, the representation space $\operatorname{Hom}\left(F_{N}, G\right)$ is simply the product group $G^{N}$, which is naturally connected as $G$ is itself connected. Indeed when one tries to apply Milnor's algorithm to the presentation above, we get that the element of $\pi_{1}(G)$ obtained, depends on the choice of the lifts of the image of the $m$ generators $C_{1}, \ldots, C_{m}$. So in 
order to have a non-trivial topological invariant, we need to be able to choose canonical lifts for the image of these generators.

An element $g$ in $G$ will have a canonical lift in $\widetilde{G}$ if it has a fixed point on $\partial \mathbb{H} \simeq \mathbb{S}^{1}$. The canonical lift, denoted $\widetilde{g}^{0}$, is the unique lift of $g$ in $\widetilde{G}$ that also has fixed points on $\widetilde{\mathbb{S}^{1}} \simeq \mathbb{R}$. By the classification of hyperbolic isometries, an isometry has a fixed point if and only if it is not elliptic. The canonical lift of a non-elliptic element will belong to $\{I\} \cup \mathcal{H}_{0} \cup \mathcal{P}_{0}$.

Now, we can define an invariant on the set

$$
W(\Sigma)=\left\{\phi \in \operatorname{Hom}\left(\pi_{1}(\Sigma), G\right) \mid \forall C_{i} \subset \partial \Sigma, \phi\left(C_{i}\right) \text { is not elliptic }\right\} .
$$

Let $\phi$ be an element of $W(\Sigma)$. Choose arbitrary lifts $\widetilde{\phi\left(A_{1}\right)}, \ldots, \widetilde{\phi\left(B_{g}\right)}$ of the images of the generators into $\widetilde{G}$. The relation of the fundamental group implies that the element

$$
\widetilde{R}=\left[\widetilde{\phi\left(X_{1}\right)}, \widetilde{\phi\left(Y_{1}\right)}\right] \ldots\left[\widetilde{\phi\left(X_{g}\right)}, \widetilde{\phi\left(Y_{g}\right)}\right] \cdot{\widetilde{\phi\left(C_{1}\right)}}^{0} \cdots{\widetilde{\phi\left(C_{m}\right)}}^{0}
$$

is a lift of the identity element of $G$ into $\widetilde{G}$ that is independent of the chosen lifts. So there exists $n \in \mathbb{Z}$ such that $\widetilde{R}=z^{n}$. We define $o_{2}(\phi)=$ $n$ and call it the relative second obstruction class of the representation $\phi$. This defines the map $o_{2}: W(\Sigma) \longrightarrow \mathbb{Z}$

The following theorem is the generalization of Theorem 2

Theorem 5. Let $S$ be an orientable surface with $\chi(S)<0$. For all $n \in \mathbb{Z}$, if $|n| \leq|\chi(S)|$ then $o_{2}^{-1}(n)$ is non-empty and connected.

As a consequence, the space $W(\Sigma)$ has $2|\chi(\Sigma)|+1$ connected components. We can see that Theorem 2 is just a particular case of Theorem 5 , when $\Sigma$ has no boundaries.

3.4. Summary of the proof. The proof of this theorem goes by induction. First, we prove the theorem for surface of Euler characteristic -1 , namely the pair-of-pants $\Sigma_{(0,3)}$ and the one-holed torus $\Sigma_{(1,1)}$. This is mostly a consequence of Propositions 1 and 2, that can describe the set $W(\Sigma)$.

Next, we decompose any orientable surface into surfaces of characteristic -1 . We want the graph dual to this decomposition to be a tree so that the induction process works. For the surface $\Sigma_{(g, m)}$, such a decomposition consists of $g$ one-holed tori and $g+m-2$ pair-of-pants. One can find a continuous path from any representation to a representation such that the curves defining the decomposition are sent to hyperbolic elements. So it is possible to compute the relative Euler class on each subsurface of the decomposition. Then, using the result 
on $\Sigma_{(0,3)}$ and $\Sigma_{(1,1)}$, one can show that if two representations have their relative Euler class coincide on each subsurface, then they are in the same connected component.

Then it suffices to show that if two representations have the same Euler class, we can find paths to representations such that the relative Euler classes coincide on each subsurface. First, one can prove this fact for each surface obtained by gluing two surfaces of Euler characteristic -1 , namely $\Sigma_{2}, \Sigma_{(1,2)}$ and $\Sigma_{(0,4)}$. Using this, one can prove the result on a general surface by induction along the tree corresponding to the dual graph of the decomposition (see [7] for a complete proof of these arguments).

\section{NON-ORIENTABLE SURFACES}

In this section, we extend the definition of the obstruction invariant to the representation of the fundamental group of a non-orientable surface into $\operatorname{PSL}(2, \mathbb{R})$, and then in $\operatorname{PGL}(2, \mathbb{R})$. We denote $G_{+}=$ $\operatorname{PSL}(2, \mathbb{R})$ and $G_{ \pm}=\operatorname{PGL}(2, \mathbb{R})$. So the group $G_{ \pm}$is the disjoint union $G_{+} \sqcup G_{-}$, where $G_{-}$is the set of orientation-reversing isometries of $\mathbb{H}^{2}$.

4.1. Non-orientable surface group. Recall that a closed non-orientable surface $N_{k}$ is homeomorphic to the connected sum of $k$ projective planes. The number $k$ is called the non-orientable genus of the surface and the Euler characteristic is $\chi\left(N_{k}\right)=2-k$.

The fundamental group is given by the following presentation :

$$
\pi_{1}\left(N_{k}\right)=\left\langle A_{1}, \ldots, A_{k} \mid A_{1}^{2} \cdots A_{k}^{2}\right\rangle .
$$

In this presentation, the generators are represented by one-sided curves, which are curves whose neighborhoods are Mobiüs strips. In particular, when $N_{k}$ is given an hyperbolic structure on $N_{k}$, the holonomy of a one-sided curve is sent to an orientation-reversing isometry.

4.2. Second obstruction class. Let us first understand the connected components of $\operatorname{Hom}\left(\pi, G_{+}\right)$, where $\pi=\pi_{1}\left(N_{k}\right)$. Let $\phi \in \operatorname{Hom}\left(\pi, G_{+}\right)$. The relation defining the fundamental group implies that the element $Q_{k}\left(A_{1}, \ldots, A_{k}\right)$ defined in Section 2 is a lift of the identity in $G_{+}$to $\widehat{G}$. This defines a map into the group $Z(\widehat{G})=\{ \pm I\} \simeq \mathbb{Z} / 2 \mathbb{Z}$. So we have the following map :

$$
o_{2}: \operatorname{Hom}\left(\pi, G_{+}\right) \rightarrow \mathbb{Z} / 2 \mathbb{Z} .
$$

As the map $Q_{k}$ is continuous, the map $o_{2}$ is also continuous and takes values in a discrete set, so we can infer that $O_{2}$ is constant on connected components of the representation space. 
We can also generalize the construction to non-orientable surfaces with boundaries, denoted $N_{(k, m)}$. As in the orientable case, we have to impose the condition that boundary components are sent to non-elliptic elements, so we define

$$
W\left(N_{(k, m)}\right)=\left\{\phi \in \operatorname{Hom}\left(\pi, G_{+}\right) \mid \forall C \in \partial N_{(k, m)}, \phi(C) \text { is not elliptic }\right\} .
$$

The map $o_{2}: W\left(N_{(k, m)}\right) \rightarrow \mathbb{Z} / 2 \mathbb{Z}$ is defined as in the case of orientable surfaces. The characteristic class $\mathrm{O}_{2}$ is sufficient to distinguish the connected components of $W(N)$, and we can state the following :

Theorem 6. Let $N_{(k, m)}$ be a non-orientable surface with $\chi\left(N_{(k, m)}\right)<$ 0 . For $[n] \in \mathbb{Z} / 2 \mathbb{Z}$, the set $o_{2}^{-1}([n]) \subset W\left(N_{(k, m)}\right)$ is non-empty and connected.

This establishes that the representation space $\operatorname{Hom}\left(\pi_{1}\left(N_{k}\right), G_{+}\right)$has exactly 2 connected components.

It may seem surprising that in the non-orientable case, the number of connected components does not depend on the non-orientable genus. In fact, representations in $G_{+}$do not parametrize hyperbolic structures on the surfaces or oriented circle bundles over the surface. Indeed, if a representation $\phi: \operatorname{Hom}\left(\pi_{1}(N), G_{ \pm}\right)$defines an oriented circle bundle over $N$, then necessarily if $A \in \pi_{1}(N)$ is represented by a one-sided curve, then $\phi(A) \in G_{-}$. Hence we have to study representations in $G_{ \pm}$

4.3. Non-orientable Euler class. The group $G_{ \pm}$is not connected anymore as $G_{ \pm}=G_{+} \sqcup G_{-}$, hence $\pi_{0}\left(G_{ \pm}\right) \simeq \mathbb{Z} / 2 \mathbb{Z}$. When studying the representation into $G_{ \pm}$, one has to use another topological invariant for the representation, namely the first obstruction class denoted $o_{1}$ and defined as follows.

The obstruction class $o_{1}(\phi)$ of a representation $\phi \in \operatorname{Hom}\left(\pi, G_{ \pm}\right)$is an element of the set $\operatorname{Hom}\left(\pi, \pi_{0}\left(G_{ \pm}\right)\right)$and is obtained by composition of the representation $\phi: \pi \rightarrow G_{ \pm}$with the epimorphism $G_{ \pm} \rightarrow \pi_{0}\left(G_{ \pm}\right)$. As $\pi$ is finitely generated by $\left\{A_{1}, \ldots, A_{k}\right\}$, the image of the generators by the map $o_{1}(\phi)$ completely determines $o_{1}(\phi)$. So we can see $\operatorname{Hom}\left(\pi, \pi_{0}\left(G_{ \pm}\right)\right)$as a subset of $\left(\pi_{0}\left(G_{ \pm}\right)\right)^{k} \simeq(\mathbb{Z} / 2 \mathbb{Z})^{k}$. We have the following notation for the first obstruction :

$$
o_{1}: \operatorname{Hom}\left(\pi_{1}\left(N_{k}\right), G_{ \pm}\right) \rightarrow(\mathbb{Z} / 2 \mathbb{Z})^{k} .
$$

For example the representation space $\operatorname{Hom}\left(\pi, G_{+}\right)$is exactly the set of representations $\phi \in \operatorname{Hom}\left(\pi, G_{ \pm}\right)$such that $o_{1}(\phi)=(0, \ldots, 0)$.

On the other hand, given a hyperbolic structure $\phi$ on $N$, the holonomy along a 1-sided curve is necessarily an orientation-reversing isometry as the curve reverse the orientation on the surface. Hence, 
the image of the 1-sided curves are all orientation-reversing isometries. The generators in the standard presentation are represented by 1-sided curves, so any holonomy representation of a hyperbolic structure satisfies $o_{1}(\phi)=(1, \ldots, 1)$.

4.4. Square map. To understand the representations inside the nonzero components $o_{1}^{-1}\left(\varepsilon_{1}, \ldots, \varepsilon_{k}\right)$, we need to understand squares of orientation-reversing isometries. First, we notice that if $A \in G_{-}$, then $A^{2} \in G_{+}$. Moreover, all orientation-reversing isometries are glidereflections, i.e. the product of a reflection and a hyperbolic translation along the same axis. Hence, the square $A^{2}$ of a glide-reflection is an hyperbolic element or the identity element. Simple matrix calculation (see [15]) also shows that the square map :

$$
\begin{aligned}
G_{-} & \longrightarrow \mathcal{H} \cup\{I\} \\
A & \longmapsto A^{2}
\end{aligned}
$$

is continuous, surjective with connected fibers.

Let $\varepsilon=\left(\varepsilon_{1}, \ldots, \varepsilon_{k}\right) \in(\mathbb{Z} / 2 \mathbb{Z})^{k}$ and define $l$ as the number of $\varepsilon_{i}$ that are zero. The fundamental group $\pi_{1}\left(N_{(l, k-l)}\right)$ can be seen as a subgroup of $\pi_{1}\left(N_{k}\right)$ by cutting the surface along the curve $A_{i}^{2}$ whenever $\varepsilon_{i} \neq 0$.

Using this property we can define a map :

$$
\begin{aligned}
T_{\varepsilon}: o_{1}^{-1}(\varepsilon) & \longrightarrow W\left(N_{l, n}\right) \\
\phi & \longmapsto T_{\varepsilon}(\phi),
\end{aligned}
$$

where $T_{\varepsilon}(\phi)\left(A_{i}\right)=\phi\left(A_{i}\right)$ if $\varepsilon_{i}=0$ and else $T_{\varepsilon}(\phi)\left(A_{i}\right)=\phi\left(A_{i}\right)^{2}$. This map is continuous, surjective with connected fibers because the square map defined above is. We infer that $o_{1}^{-1}\left(\varepsilon_{1}, \ldots, \varepsilon_{k}\right)$ and $W\left(N_{(l, n)}\right)$ have the same number of connected components.

For all $\varepsilon$ such that $l>0$, Theorem 6 asserts that $W\left(N_{(l, n)}\right)$ has two connected components indexed by $o_{2} \in \mathbb{Z} / 2 \mathbb{Z}$. However, when $l=0$, we identify $N_{(0, k)}=\Sigma_{(0, k)}$ as the n-holed sphere. In this case, Theorem 5 tells us that $\left.W\left(\Sigma_{(0, k}\right)\right)$ has $2 k-3$ connected components indexed by the relative obstruction class $O_{2} \in \mathbb{Z}$.

We can now state the following theorem on the total number of connected component of $\operatorname{Hom}\left(\pi, G_{ \pm}\right)$.

Theorem 7 . The representation space $\operatorname{Hom}\left(\pi_{1}\left(N_{k}\right), \operatorname{PGL}(2, \mathbb{R})\right)$ has $2^{k+1}+2 k-5$ connected components.

The set of representations such that $l=0$ is the fiber $o_{1}^{-1}(1, \ldots, 1)$. Through the map $T_{\varepsilon}$ we can define the obstruction of a representation $\phi \in o_{1}^{-1}(1, \ldots, 1)$ as the relative obstruction class of $T_{\varepsilon}(\phi) \in W\left(\Sigma_{(0, k)}\right)$. It can be shown that this invariant coincides with the Euler class of 
the oriented circle bundle over $N_{k}$ defined by $\phi$. The components of $\operatorname{Hom}\left(\pi, G_{ \pm}\right)$consisting of discrete and faithful representations are exactly the ones with $\varepsilon=(1, \ldots, 1)$ and maximal Euler class.

4.5. General Formula. In [18], Xia proves that for an orientable surface of genus $g$, the space $\operatorname{Hom}\left(\pi_{1}\left(\Sigma_{g}\right), G_{ \pm}\right)$has $2^{2 g+1}+4 g-5$ connected components. When translating the formula in terms of the Euler characteristic of the surface, one obtain the following general result.

Theorem 8. Let $S$ be a closed surface orientable or not, with Euler characteristic $\chi(S)<0$ and $G_{ \pm}=\operatorname{PGL}(2, \mathbb{R})$, then the number $N(S)$ of connected components of the space $\operatorname{Hom}\left(\pi_{1}(S), G_{ \pm}\right)$is given by :

$$
N(S)=2^{3-\chi(S)}-2 \chi(S)-1 .
$$

\section{ACKNOWLEDGEMENTS}

The author would like to thank all the organizers for the very interesting and enriching program. This paper was written while the author was a CIRGET postdoctoral fellow at the Université de Sherbrooke.

\section{REFERENCES}

[1] Bradlow, S.B., Garca-Prada, O., Gothen, P.B.: Maximal surface group representations in isometry groups of classical Hermitian symmetric spaces, Geom. Dedicata 1222006 , 185-213

[2] Burger, M., Iozzi, A., Labourie, F., Wienhard, A.: Maximal representations of surface groups:Symplectic Anosov structures, Pure Appl. Math. Q. 13 (2005), $543-590$

[3] Burger, M., Iozzi, A., Wienhard, A.: Higher Teichmüller spaces : From $\mathrm{SL}(2, \mathbb{R})$ to other Lie groups, Preprint, arXiv:1004.2894v2

[4] Choi, S., Goldman W.: The classification of real projective structures on compact surfaces., Bull. Amer. Math. Soc. 34 (1997), no. 2, 161-171.

[5] Dumas, D. Complex projective structures, Handbook of Teichmller theory. Vol. II, 455-508.

[6] Garcia-Prada, O., Gothen, P.B., Mundet i Riera, I.: Representations of surface groups in $S p(2 n, R)$, arXiv:0809.0576 (September 2008)

[7] Goldman, W.: Topological components of spaces of representations, Invent. Math. 93 (1988), 557-607.

[8] Goldman W., Mapping class group dynamics on surface group representation in "Problems on Mapping Class Groups and Related Topics", B. Farb, ed. Proc. Symp. in Pure Math. 74, American Mathematical Society (2006), 189-214

[9] Goldman W., Locally Homogeneous Geometric Structures Proceedings of the 2010 International Congress of Mathematicians, Hyderabad, India (2010), 717744.

[10] Hitchin, N.J.: Lie groups and Teichmüller space, Topology 31 (1992), 449-473.

[11] Jankins, M., Neumann, W.: Homomorphisms of fuchsian groups to $\operatorname{PSL}(2, \mathbb{R})$, Comment. Math. Helvetici, 60 (1985), 480-495. 
[12] Milnor, J.: On the existence of a connection with zero curvature, Comment. Math. Helv. 32 (1958), 215-223.

[13] Wood, J.: Bundles with totally disconnected structure group, Comment. Math. Helv. 46 (1971), 257-273.

[14] Palesi, F.: Ergodic action of the mapping class group on moduli spaces of representation of non-orientable surfaces Geometriae Dedicata 151 (2011), 107$140)$.

[15] Palesi, F.: Connected components of representation spaces of non-orientable surfaces Communications in Analysis and Geometry, 18 (2010), 195-21.

[16] Palesi, F.: Non-Teichmüller components for non-orientable surfaces in preparation.

[17] Toledo, D.: Representations of surface groups in complex hyperbolic space, J. Differential Geom. 29 (1989), 125-133.

[18] Xia, E.Z.: Components of $\operatorname{Hom}\left(\pi_{1}, \mathrm{PGL}(2, \mathbb{R})\right)$, Topology 36 (1997), 481-499.

Département de Mathématiques, Université de Sherbrooke, SherBROOKE, QC J1H4C6, CANADA, FREDERIC.PALESI@USHERBROOKE.CA 